PROCEEDINGS OF THE

AMERICAN MATHEMATICAL SOCIETY

Volume 125, Number 9, September 1997, Pages 2637-2642

S 0002-9939(97)03857-4

\title{
CELLULAR FILTRATION OF K-THEORY AND DETERMINANTS OF $C^{*}$-ALGEBRAS
}

\author{
LIANGQING LI
}

(Communicated by Palle E. T. Jorgensen)

\begin{abstract}
In this note, we will disprove the following conjecture raised by Exel-Loring: Let $A$ be a $C^{*}$-algebra with trace $\tau$ and let det $: U_{\infty} \rightarrow$ $\mathbb{R} / \tau_{*}\left(K_{0}(A)\right)$ be a determinant associated to $\tau$. If $\phi_{t}: C\left(S^{3}\right) \rightarrow A(0 \leq t \leq 1)$ is a continuous family of homomorphisms and $b \in C\left(S^{3}\right) \otimes M_{2}$ is the canonical matrix valued function on $S^{3}$ which represents the Bott element in $K_{1}\left(C\left(S^{3}\right)\right)$, then $\operatorname{det}\left(\phi_{0}(b)\right)=\operatorname{det}\left(\phi_{1}(b)\right)$. It should be noticed that the conjecture has been proved by Exel-Loring for the case that $\phi_{t}$ is a smooth family of homomorphisms.
\end{abstract}

Let $b_{n}$, or simply $b$, denote the element of $K_{*}\left(C\left(S^{n}\right)\right)(n \geq 1)$ which has the Chern character equal to the fundamental class (i.e., the canonical generator of $\left.H^{n}\left(S^{n}\right)\right)$. We call $b$ the Bott element. In [ExL], Exel-Loring tried to extend the concept of cohomology of topological spaces (commutative $C^{*}$-algebras) to general $C^{*}$-algebras and define a filtration of $K_{*}(A)$ of any $C^{*}$-algebra $A$ as the following:

Definition 1. Let $A$ be a unital $C^{*}$-algebra. The spherical filtration $F_{*} K_{*}(A)$ of $K_{*}(A)$ is defined by

$$
\begin{aligned}
& F_{0} K_{0}(A)=K_{0}(A), \\
& F_{1} K_{1}(A)=K_{1}(A), \\
& F_{n} K_{n}(A)=\left\{x \in K_{n}(A) ; x=\phi_{*}(b) \text { for some } \phi \in \operatorname{Hom}\left(C\left(S^{n}\right), M_{k} \otimes A\right)\right\} .
\end{aligned}
$$

Exel-Loring proved that the cohomology $H^{n}(X) \otimes \mathbb{Q}$ with rational coefficient of finite CW complex $X$ can be recovered from the filtration of $K_{*}(C(X))$ by

$$
H^{n}(X) \otimes \mathbb{Q}=\frac{\left(F_{n} K_{*}(C(X))\right) \otimes \mathbb{Q}}{\left(F_{n+2} K_{*}(C(X))\right) \otimes \mathbb{Q}} .
$$

Also, they defined

$$
H_{n}(A)=\frac{F_{n} K_{*}(A)}{F_{n+2} K_{*}(A)} .
$$

It is easy to see that, for any $C^{*}$-algebra $A$,

$$
F_{2} K_{*}(A) \subset \bigcap_{\tau \in T A} \operatorname{Ker} \tau_{*},
$$

Received by the editors January 22, 1996 and, in revised form, March 18, 1996 1991 Mathematics Subject Classification. Primary 46L80, 46M20, 19 K56.

(C)1997 American Mathematical Society 
where $T A$ is the set of traces on $A$, and the map $\tau_{*}: K_{0}(A) \rightarrow \mathbb{R}$ is induced by $\tau$. In [EL], it is proved that for many AF algebras $A$ without finite quotient,

$$
F_{2} K_{*}(A)=\bigcap_{\tau \in T A} \operatorname{Ker} \tau_{*} .
$$

It can also be proved, by the the strategy used in the present paper, that for many $\mathrm{AF}$ algebras and other real rank zero $C^{*}$-algebras $A$,

$$
F_{2} K_{*}(A)=F_{2 n} K_{*}(A) \quad \text { for } \quad n \geq 1 .
$$

It seems that, in non-commutative $C^{*}$-algebras, the only efficient tool to calculate the filtration $F_{2 n} K_{0}(A)$ of $K_{0}(A)$ is the traces. A natural problem is to find some analogue tool for computing the filtration $F_{2 n+1} K_{1}(A)$ of $K_{1}(A)$. Motivated by this problem, Exel and Loring were trying to consider the determinants of $C^{*}$ algebras as a tool and relate homomorphisms to determinants. In order for the determinants to be an efficient tool, they proposed some conjectures. And based on these conjectures, they proved several theorems. We briefly review some definitions and results in $[\mathrm{ExL}]$.

We use $U_{k}(A)$ to denote the set of unitaries in $M_{k}(A)$ and denote $U_{\infty}(A) \triangleq$ $\bigcup_{k=1}^{\infty} U_{k}(A)$ by embedding

$$
a \rightarrow\left(\begin{array}{cc}
a & 0 \\
0 & 1
\end{array}\right)
$$

Considering $S^{3}$ as the unit sphere in $\mathbb{C}^{2}$, let $b \in U_{2}\left(C\left(S^{3}\right)\right)$ be defined by

$$
b(m, n)=\left(\begin{array}{cc}
m & -\bar{n} \\
n & \bar{m}
\end{array}\right) \in U_{2}\left(C\left(S^{3}\right)\right) \quad \text { for }(m, n) \in S^{3} .
$$

It is known that $b$ represents the generator in $K_{1}\left(C\left(S^{3}\right)\right)$ (i.e., the Bott element).

Let $A$ be a $C^{*}$-algebra with a trace $\tau$, and the map $\tau_{*}: K_{0}(A) \rightarrow \mathbb{R}$ be induced by $\tau$. Let

$$
q: \mathbb{R} \longrightarrow \mathbb{R} / \tau_{*}\left(K_{0}(A)\right)
$$

denote the canonical quotient map.

Definition 2. We say that a group homomorphism

$$
\operatorname{det}: U_{\infty}(A) \longrightarrow \mathbb{R} / \tau_{*}\left(K_{0}(A)\right)
$$

is a determinant function associated to the trace $\tau$ on the $C^{*}$-algebra $A$ if

$$
\operatorname{det}\left(e^{2 \pi i h}\right)=q(\tau(h)), \quad h \in M_{n}(A) .
$$

We will also say that det is a determinant associated to $(A, \tau)$.

A determinant associated to a trace is not unique, except on the connected component of the identity. However, determinants always exist, and any two determinants are related in a simple way (see $\S 7$ of $[\mathrm{Ex}]$ ).

In $[E x L]$, several conjectures are proposed. One of them is the following:

Conjecture $1(\S 7.5$ in $[\mathrm{ExL}])$. Suppose that det is a determinant associated to $(A, \tau)$. If $\phi: C\left(S^{3}\right) \rightarrow A$ is a homomorphism, then $\operatorname{det}(\phi(b))$ depends only on $\phi_{*}(b) \in K_{1}(A)$. In particular, if $\phi_{*}(b)=0$, then $\operatorname{det}(\phi(b))=0$. 
In [ExL], this conjecture was proved for the case of $A=M_{k}(C(X)$ ). (See 7.4 of $[\mathrm{ExL}]$.

In $[\mathrm{ExL}]$, the following conjecture was also proposed which is weaker than the above one.

Conjecture 2 ( $\$ 7.6$ in $[\mathrm{ExL}])$. Suppose that det is a determinant associated to $(A, \tau)$. If $\phi_{t}: C\left(S^{3}\right) \rightarrow A$ is a continuous path of homomorphisms, then $\operatorname{det} \phi_{0}(b)=$ $\operatorname{det}\left(\phi_{1}(b)\right)$.

This conjecture was proved for the case of $\phi_{t}: C\left(S^{3}\right) \rightarrow A$ being a smooth path of homomorphisms (i.e., the two maps $t \mapsto \phi_{t}(m), t \mapsto \phi_{t}(n)$ are smooth) in [ExL].

Applying Conjecture 2, Exel and Loring proved several results about cellular cohomology.

In this note, we will construct a counterexample for Conjecture 2, and therefore disprove both conjectures. The result reveals some significant differences between non commutative topology and commutative topology - it is known that a continuous homotopy of two maps from a topological space $X$ to a smooth manifold $M$ can always be replaced by a smooth path of homotopy of these two maps, i.e., by a homotopy $F_{t}: X \times[0,1] \rightarrow M$ which is smooth with respect to the parameter $t$. The proof of our result involves some deep classification theory of $C^{*}$-algebras of Elliott in [El].

Our counterexample is a Bunce-Dedden algebra constructed as follows. Set

$$
D=\left\{e^{2 \pi \frac{k}{2^{n}} i} ; k, n \text { are arbitrary integers }\right\} .
$$

The $D$ is a subgroup of $S^{1} \subset \mathbb{C}$. Let $\alpha$ be the action of $D$ on $C\left(S^{1}\right)$ by rotation, i.e.,

$$
\alpha_{e^{2 \pi \frac{k}{2^{n} i}}(f)(z)=f\left(e^{2 \pi \frac{k}{2^{n}} i} \cdot z\right)}
$$

for any $f \in C\left(S^{1}\right)$ and $z \in S^{1}$. Bunce-Dedden algebra $B D$ is defined by

$$
B D=C\left(S^{1}\right) \rtimes_{\alpha} D .
$$

It is known (see [BBEK], for example) that $B D$ is an inductive limit of matrix algebras over $C\left(S^{1}\right)$, and $B D$ is also of real rank zero (i.e., the set of invertible selfadjoint elements is dense in the set of selfadjoint elements). Furthermore,

$$
K_{0}(B D)=\mathbb{Z}\left(\frac{1}{2}\right), \quad K_{1}(B D)=\mathbb{Z},
$$

where

$$
\mathbb{Z}\left(\frac{1}{2}\right)=\left\{\frac{k}{2^{n}} ; k, n \text { are integers }\right\} \subset \mathbb{R} .
$$

For our purpose, we need to introduce the positive cone $K_{*}(A)_{+}$of $K_{*}(A)$ (see [El]) and the scale $\mathbf{1}_{A} \in K_{*}(A)$ for a $C^{*}$-algebra $A$. First, $\mathbf{1}_{A}$ is the element in $K_{0}(A) \subset K_{*}(A)$ corresponding to the unit of $A$. And $K_{*}(A)_{+}$is defined by

$$
K_{*}(A)_{+}=\left\{([p],[u+(1-p)]) \in K_{0}(A) \oplus K_{1}(A) ; p, u \in M_{k}(A), u u^{*}=u^{*} u=p\right\} .
$$

It is also known that $K_{*}(B D)_{+} \backslash\{0\}=\left(\mathbb{Z}\left(\frac{1}{2}\right)_{+} \backslash\{0\}\right) \oplus \mathbb{Z}$ and $\mathbf{1}_{B D}=(1,0) \in$ $K_{0}(A) \oplus K_{1}(A)$.

The following two theorems prove that $B D$ is a counterexample of Conjecture 2 .

Theorem 1. $F_{3} K_{1}(B D)=\mathbb{Z}$. 
Theorem 2. If Conjecture 2 is true for the $C^{*}$-algebra $B D$, then $F_{3} K_{1}(B D)=0$.

In the proof of Theorem 1, we need the following lemma.

Lemma 1. Suppose that $\alpha: K_{*}(A) \otimes K_{*}(B) \rightarrow K_{*}(A \otimes B)$ is the map defined in the Kunneth formula. If $x \in F_{k} K_{*}(A)$ and $y \in F_{l} K_{*}(B)$, then $\alpha(x \otimes y) \in$ $F_{k+l} K_{*}(A \otimes B)$.

Proof. By Definition 1, there are maps $\phi: C\left(S^{k}\right) \rightarrow M_{a}(A)$ and $\psi: C\left(S^{l}\right) \rightarrow M_{b}(B)$ such that

$$
\phi_{*} b_{k}=x \quad \text { and } \quad \psi_{*} b_{l}=y .
$$

We can identify $K_{*}\left(C\left(S^{k}\right)\right) \otimes K_{*}\left(C\left(S^{l}\right)\right)$ with $K_{*}\left(C\left(S^{k}\right) \otimes C\left(S^{l}\right)\right)$ via the Kunneth formula. Hence

$$
b_{k} \otimes b_{l} \in K_{*}\left(C\left(S^{k}\right) \otimes C\left(S^{l}\right)\right)=K_{*}\left(C\left(S^{k} \times S^{l}\right)\right) .
$$

Also, it is well known, from the definition of tensor product (or cup product) of cohomologies of topological spaces, that $b_{k} \otimes b_{l}$ is in $H^{k+l}\left(S^{k} \times S^{l}\right)$, via the Chern map. That is,

$$
b_{k} \otimes b_{l} \in F_{k+l} K_{*}\left(C\left(S^{k}\right) \otimes C\left(S^{l}\right)\right) .
$$

Consider the map $\phi \otimes \psi: C\left(S^{k} \times S^{l}\right) \rightarrow M_{a b}(A \otimes B)$. Since the exact sequence of the Kunneth formula is natural,

$$
(\phi \otimes \psi)_{*}\left(b_{k} \otimes b_{l}\right)=\alpha(x \otimes y) .
$$

This proves

$$
\alpha(x \otimes y) \in F_{k+l} K_{*}(A \otimes B) .
$$

Proof of Theorem 1. Let $A$ be the AF algebra with $K_{0}(A)=\mathbb{Z}\left(\frac{1}{2}\right) \oplus \mathbb{Z}, K_{0}(A)_{+} \backslash\{0\}$ $=\left(\mathbb{Z}\left(\frac{1}{2}\right)_{+} \backslash\{0\}\right) \oplus \mathbb{Z}$, and $\mathbf{1}_{A}=(1,0) \in K_{0}(A)$. Then by [EL], there is a homomorphism $\psi: C\left(T^{2}\right) \rightarrow A$ such that $\psi_{*}(x)=(0,1) \in \mathbb{Z}\left(\frac{1}{2}\right) \oplus \mathbb{Z}=K_{0}(A)$, where $x \in K_{0}\left(C\left(T^{2}\right)\right)$ is the generator of $H^{2}\left(T^{2}\right)$. This proves that $F_{2}\left(K_{0}(A)\right)=\mathbb{Z}$. Considering $A \otimes B D$, we know that, by the Kunneth formula,

$$
K_{0}(A \otimes B D)=\mathbb{Z}\left(\frac{1}{2}\right) \oplus \mathbb{Z}\left(\frac{1}{2}\right), \quad K_{1}(A \otimes B D)=\mathbb{Z}\left(\frac{1}{2}\right) \oplus \mathbb{Z} .
$$

Furthermore, by Lemma 1 , the $\mathbb{Z}$ summand of $K_{1}(A \otimes B D)$ is in $F_{3} K_{1}(A \otimes B D)$ (notice that it comes from the tensor product of $\mathbb{Z}=F_{2} K_{0}(A)$ and $\mathbb{Z}=K_{1}(B D)$ ). We also have

$$
K_{*}(A \otimes B D)_{+} \backslash\{0\}=\left(\left(\mathbb{Z}\left(\frac{1}{2}\right)_{+} \backslash\{0\}\right) \oplus \mathbb{Z}\left(\frac{1}{2}\right)\right) \oplus\left(\mathbb{Z}\left(\frac{1}{2}\right) \oplus \mathbb{Z}\right)
$$

and

$$
\mathbf{1}_{A \otimes B D}=(1,0,0,0) \in K_{0}(A \otimes B D) \oplus K_{1}(A \otimes B D) .
$$

Let $\beta: K_{*}(A \otimes B D) \rightarrow K_{*}(B D)$ be defined by

$$
\beta(a, b, c, d)=(a, d) \in K_{0}(B D) \oplus K_{1}(B D)=K_{*}(B D) .
$$


Then $\beta$ is a graded homomorphism which preserves the order and the scale. By the Elliott classification theory (see 7.3 of $[\mathrm{El}]$ ), there is a homomorphism $\phi: A \otimes B D \rightarrow$ $B D$ such that $\phi_{*}=\beta$. Therefore, we have proved that

$$
\mathbb{Z} \subset \phi_{*} F_{3} K_{1}(A \otimes B D) \subset F_{3} K_{1}(B D)=\mathbb{Z} .
$$

This ends the proof of Theorem 1 .

Proof of Theorem 2. The proof is similar to that of Proposition 7.8 in [ExL]. Let $u$ be the canonical unitary generator of $C\left(S^{1}\right) \subset C\left(S^{1}\right) \rtimes_{\alpha} D$, and $\gamma$ be the action on $B D$ defined by

$$
\gamma(u)=e^{2 \pi i \sqrt{2}} u ; \quad \gamma(g)=g \quad \text { for } \quad g \in D .
$$

Since $\gamma$ is homotopic to the identity homomorphism, one has that $\gamma_{*}=$ id on $K_{1}(B D)$. Let $\tau$ be the canonical trace on $B D$, i.e.,

$$
\tau\left(\sum_{d \in D} f_{d}[d]\right)=\int_{S^{1}} f_{0} d \theta
$$

for any $\sum_{d \in D} f_{d}[d] \in C\left(S^{1}\right) \rtimes_{\alpha} D$. Define the rotation number map

$$
\rho_{\gamma}^{\tau}: K_{1}(B D) \longrightarrow \mathbb{R} / \tau_{*}\left(K_{0}(B D)\right)
$$

of $\gamma$ by

$$
\rho_{\gamma}^{\tau}([v])=\operatorname{det}\left(\gamma\left(v^{*}\right) v\right)
$$

(see $[\mathrm{ExL}]$ and $[\mathrm{Ex}]$ for details about $\rho_{\gamma}^{\tau}$.) It is easy to see that

$$
\rho_{\gamma}^{\tau}([u])=\operatorname{det}\left(\gamma\left(u^{*}\right) u\right)=\sqrt{2} \notin \mathbb{Z}\left(\frac{1}{2}\right)=\tau_{*}\left(K_{0}(B D)\right)
$$

for the generator $[u] \in K_{1}(B D)$. Hence $\rho_{\gamma}^{\tau}: K_{1}(B D) \rightarrow \mathbb{R} / \tau_{*}\left(K_{0}(B D)\right)$ is injective.

Suppose that we are given $\phi: C\left(S^{3}\right) \rightarrow M_{k} \otimes B D$. Then $\phi$ and $\gamma \circ \phi$ are homotopic. So by the conjecture,

$$
\rho_{\gamma}^{\tau}([\phi(b)])=-\operatorname{det}(\gamma \circ \phi(b))+\operatorname{det}(\phi(b))=0 .
$$

Hence $\phi_{*}(b)=0$ in $K_{1}(B D)$.

Combining Theorem 1 and Theorem 2, we disproved Conjecture 2.

Inspired by this paper, the second counterexample of the conjecture was discovered by Elliott-Gong [EG]. Their example involves a deep structure theory of the irrational rotation algebras, and the reduction of inductive limits of matrix algebras over $C\left(T^{2}\right)$ to that over $C\left(S^{1}\right)$.

\section{REFERENCES}

[BBEK] B. Blackadar, O. Bratteli, G. Elliott, and Kumjian, A., Reduction of real rank in inductive limits of $C^{*}$-algebras, Math. Ann. 292 (1992), 111-126. MR 93a:46112

[El] G.A. Elliott, On the classification of $C^{*}$-algebras of real rank zero, J. Reine Angew. Math. 443 (1993), 179-219. MR 94i:46074

[EG] G. Elliott and G. Gong, On inductive limits of matrix algebras over two tori, Amer. J. of Math. 118 (1996), 263-290.

[EL] G. Elliott and T. Loring, AF embedding of $C\left(T^{2}\right)$ with prescribed $K$ theory, J. of Functional Analysis 103 (1992), 1-25. MR 93b:46134 
[Ex] R. Exel, Rotation numbers for automorphisms of $C^{*}$-algebras, Pasific J. of Math 127 (1987), 31-89. MR 88a:46068

[ExL] R. Exel and T. Loring, Extending cellular cohomology to, Tran. of AMS 329 (Jan. 1992), 141-160. MR 92e:46137

The Fields Institute, 222 College Street, Toronto, Ontario, Canada M5T 3J1

E-mail address: li@fields.utoronto.ca

Current address: Department of Mathematics, University of Puerto Rico, Rio Piedras, P. O. Box 23355, San Juan, Puerto Rico 00931 